\title{
Come a long way, still a ways to go: from predicting and preventing fluoropyrimidine toxicity to increased efficacy?
}

\author{
Seid Hamzic ${ }^{1,2}$, Ursula Amstutz*,1 \& Carlo R Largiadèr ${ }^{1}$ \\ ${ }^{1}$ University Institute of Clinical Chemistry, Inselspital Bern University Hospital, University of Bern, Inselspital INO-F, CH-3010 Bern, \\ Switzerland \\ ${ }^{2}$ Graduate School for Cellular \& Biomedical Sciences, University of Bern, Freiestrasse 1, CH-3012 Bern, Switzerland \\ * Author for correspondence: ursula.amstutz@insel.ch
}
“while additional genetic factors or phenotyping approaches may complement pharmacogenetic testing in the future, DPYD genotyping provides an important tool that is available today to identify patients at increased risk of severe adverse effects from FP-based therapies."

First draft submitted: 21 March 2018; Accepted for publication: 22 March 2018; Published online: 22 May 2018

Keywords: BSA-based dosing • capecitabine $\bullet$ DPYD • pharmacogenetics • TDM • 5-FU

The understanding of the causes leading to adverse drug reactions related to the fluoropyrimidine (FP) chemotherapeutic drugs 5-fluorouracil (5-FU) and capecitabine (Cp) has improved drastically over the last decades. At first suggested as a textbook example of a pharmacogenetic syndrome but later debated as having limited clinical relevance, the evidence is now clear from a large number of prospective and retrospective studies as well as metaanalyses that enzyme activity reducing variants in the DPYD gene encoding for the rate-limiting 5-FU catabolic enzyme DPD confer an increased risk of FP-related toxicities [1]. As a result, DPYD genotyping is slowly finding its way into the clinics. In some European countries, pre-emptive genetic testing of DPYD is already a reality, or at least the analysis costs are covered by compulsory health insurance. While this progress is very encouraging, more research may further improve our understanding of FP-related adverse drug reactions, and efforts toward the global implementation of pretherapeutic testing of DPD in the clinics should continue, in order to improve the safety and efficacy of these widely used chemotherapies.

Currently, four single nucleotide polymorphisms in DPYD, c. 1905+1G>A (rs3918290, DPYD*2A), c. $1679 \mathrm{~T}>\mathrm{G}\left(\mathrm{rs} 55886062, D P Y D^{*} 13\right)$, c. $2846 \mathrm{~A}>\mathrm{T}(\mathrm{rs} 67376798)$ and c.1129-5923C $>\mathrm{A}$ (rs75017182, c.1236G $>$ A/HapB3) are the best clinically validated genetic toxicity markers in FP chemotherapy [1]. Recent research characterized the phenotypic impact of each mutation, enabling a stratification of risk variants according to their effect on enzyme function [2]. This resulted in the proposition of an enzymatic activity score, similar to that used for cytochrome P450 drug metabolizing enzymes (e.g., CYP2D6), and adjusted dosing recommendations for each score [3,4]. Furthermore, pre-emptive genetic testing was not only shown to have a benefit for the patients but also to be cost-effective [5]. So far, this evidence is available only for c.1905+1G>A. However, an ongoing clinical trial is currently evaluating the safety, feasibility and cost-effectiveness of screening for all four above-mentioned DPYD variants (ClinicalTrials.gov identifier NCT02324452).

Due to the population frequency of currently known DPYD risk variants ( $~ 5 \%$ in European populations), which is considerably lower than the frequency of FP-related early-onset toxicity (10-25\%), the above-mentioned four DPYD variants cannot account for all occurrences of severe adverse effects. About 20-30\% of FP-related toxicities can be explained by currently known DPYD risk mutations in Caucasians $[4,6]$. This sensitivity may differ in non-Caucasian populations where DPYD variation and its impact on FP-related toxicity are understudied. Indeed, additional reduced function $D P Y D$ variants have been identified in non-Caucasian populations [7]. With the increasing availability of resequencing data, the genetic characterization of DPYD has been expanded beyond well-studied candidate variants. In fact, $>450$ exonic variants in $D P Y D$ with potentially damaging effect have 
been reported [7]. However, many of these variants are very rare and have not been observed even in large cohort studies [8,9]. Given the limited possibilities of obtaining in vivo data for rare variants, both in vitro and in silico tools have been established to predict a damaging impact on DPD activity for exonic DPYD variants, which can help in the treatment of patients carrying previously uninvestigated variants [7]. While such methods are useful for exonic variants with a potential direct impact on enzyme function, they cannot investigate or predict potential regulatory effects of sequence variants. To date, the existence and potential clinical relevance of regulatory variation in DPYD is largely unknown and its investigation may lead to the discovery of additional genetic risk factors to improve the sensitivity of DPYD genotyping.

In addition, the current literature may underestimate the overall importance of $D P Y D$ variation with regard to the FP-metabolizer phenotype due to additional variability in drug exposure unrelated to interindividual differences in drug metabolism. The interindividual variability in 5-FU exposure is very high, and current practice of normalizing the dose according to body surface area (BSA) reduces this variability only by a small fraction [10]. Furthermore, significant drug exposure differences have been observed between men and women with BSA-based dosing, with higher exposure and more adverse effects in women, suggesting additional factors affecting 5-FU exposure, which may not be related to drug metabolism [11]. Indeed, interindividual and sex differences in drug exposure may arise from differences in body composition, as indicated by reports suggesting lean body mass or fat-free mass as a better predictor for dose adjustment [11]. Differences in lean body mass between men and women are well known and could at least partly explain the observed sex differences in drug exposure and toxicity [11]. This 'masking' variability in drug exposure resulting from the combination of unaccounted differences in body composition and BSA-based dosing presents a challenge for the discovery and characterization of genetic associations with an impact on drug pharmacokinetics. For example, carriers of risk variants resulting in reduced drug metabolism may have lower drug exposure due to their body composition and thus may tolerate BSA-based standard doses in spite of their reduced 5-FU clearance, while some patients without risk variants may experience toxicity through overexposure due to their body composition. Currently, genetic characterization of patients combined with data on individual FP exposure is extremely scarce. Studies jointly evaluating genetic and nongenetic predictors of exposure (e.g., related to body size and composition), may not only lead to improved dosing algorithms, but also to a better estimate of the impact of DPYD genetic variation on FP-related toxicities. This impact is currently not well known for some clinically important DPYD risk variants such as the deep intronic splice variant c.1129-5923C > A (rs75017182, HapB3). While this relatively common variant has been associated with a moderate reduction of DPD activity, its impact on 5-FU clearance in cancer patients has not been assessed [3]. With current dosing recommendations for this variant being based primarily on enzyme activity, 5-FU exposure data would be highly beneficial to refine genotype-based dosing strategies. Similarly, a germline variant in mir-27a (rs895819) was recently shown to impact DPD regulation in cell models and in peripheral blood monocytes of healthy volunteers by altering the secondary structure of this miRNA [12]. Two studies consistently observed that this variant was associated with toxicity risk in DPYD risk variant carriers [13]. However, so far, dose recommendations to enable combination of rs895819 with $D P Y D$ genotyping in a clinical context are not available due to a lack of data on the combined impact of rs895819 and DPYD genotype on 5-FU pharmacokinetics.

In addition to unaccounted variability in body composition, different studies consistently showed that most patients are underdosed with standard BSA-based dosing, and may therefore not receive the optimal therapeutic benefit of 5-FU therapy [10]. Given that intravenous 5-FU is a very good target for drug monitoring (TDM) since its area under the curve can be assessed with a single steady-state blood sample, patients receiving 5-FU infusion-based therapies would benefit from an increased use of TDM for dose adjustment. Indeed, several studies have shown that TDM-based 5-FU dosing resulted in a dose increase in a majority of patients without any increase in adverse effects and some small trials even suggested increased efficacy [14]. However, TDM is coupled to more effort and is preanalytically challenging. Successful 5-FU TDM often requires an additional clinic visit for the patients, due to the low accuracy in the run time of infusion pumps, which makes blood draws near the end of the planned infusion time unreliable. Furthermore, 5-FU is not stable in blood, which requires rapid processing of samples or the addition of a stabilizing agent. A simplification of this process would likely increase the uptake of TDM for 5-FU and thus improve dosing of 5-FU chemotherapy. In contrast to 5-FU, capecitabine and its downstream metabolites are observed only at low concentrations in plasma due to its intracellular activation, making TDM more challenging [15]. Therefore, pharmacogenetic testing may be even more relevant in $\mathrm{Cp}$-treated patients, where routine TDM currently is not feasible. In addition to the known DPYD risk variants, studies have reported variants in genes in the $\mathrm{Cp}$-activation pathway (e.g., $C D A$ and $C E S 1$ ) as promising toxicity markers specifically for $\mathrm{Cp}$-based 
therapies $[16,17]$. However, more research is needed to replicate these associations and better understand the impact of these genes and variants on Cp-related toxicities.

Besides genotyping, there have been reports on various phenotypic approaches to detect reduced DPD activity pretherapy. Most of these approaches are based on measuring endogenous uracil $(\mathrm{U})$ or its ratio with dihydrouracil $\left(\mathrm{UH}_{2}\right)$ in different body fluids, DPD activity in peripheral blood monocytes pretherapy, or a combination of phenotypic and genetic screening $[18,19]$. However, despite some promising results, it is still not fully elucidated if baseline levels of $\mathrm{U}$ and $\mathrm{UH}_{2}$ are predictive enough for systemic DPD activity and therefore have utility as toxicity markers, and if so, which phenotypic marker has the best utility. It appears that prediction of DPD activity is improved upon enzyme saturation, which can be achieved with a uracil loading dose [20]. However, this approach is time consuming and requires multiple blood samples, and may thus not be feasible for routine screening of patients.

Taken together, while additional genetic factors or phenotyping approaches may complement pharmacogenetic testing in the future, DPYD genotyping provides an important tool that is available today to identify patients at increased risk of severe adverse effects from FP-based therapies. Finally, no matter which pretherapeutic tests will eventually find their way into routine care, TDM should always complement these methods, at least for the initial cycles, for optimal FP dose individualization. This combination would not only increase the safety of FP-therapies but also holds the promise of optimizing its efficacy [14].

\section{Financial \& competing interests disclosure}

The authors acknowledge funding from the 'Swiss National Science Foundation (SNF)' under grant number 163205. The authors have no other relevant affiliations or financial involvement with any organization or entity with a financial interest in or financial conflict with the subject matter or materials discussed in the manuscript apart from those disclosed.

No writing assistance was utilized in the production of this manuscript.

\section{References}

1 Meulendijks D, Henricks LM, Sonke GS et al. Clinical relevance of DPYD variants c.1679T>G, c.1236G >A/HapB3, and c.1601G >A as predictors of severe fluoropyrimidine-associated toxicity: a systematic review and meta-analysis of individual patient data. Lancet Oncol. 16(16), 1639-1650 (2015).

2 Nie Q, Shrestha S, Tapper EE et al. Quantitative contribution of rs75017182 to dihydropyrimidine dehydrogenase mRNA splicing and enzyme activity. Clin. Pharmacol. Ther. 102(4), 662-670 (2017).

3 Henricks LM, Lunenburg CA, Meulendijks D et al. Translating DPYD genotype into DPD phenotype: using the DPYD gene activity score. Pharmacogenomics 16(11), 1275-1284 (2015).

4 Amstutz U, Henricks LM, Offer SM et al. Clinical Pharmacogenetics Implementation Consortium (CPIC) Guideline for Dihydropyrimidine Dehydrogenase Genotype and Fluoropyrimidine Dosing: 2017 Update. Clin. Pharmacol. Ther. 103(2), 210-216 (2018).

5 Deenen MJ, Meulendijks D, Cats A et al. Upfront genotyping of $D P Y D * 2 A$ to individualize fluoropyrimidine therapy: a safety and cost analysis. J. Clin. Oncol. 34(3), 227-34 (2018).

6 Amstutz U, Froehlich TK, Largiadèr CR. Dihydropyrimidine dehydrogenase gene as a major predictor of severe 5-fluorouracil toxicity. Pharmacogenomics 12, 1321-1336 (2011).

7 Shrestha S, Zhang C, Jerde CR, Hu Li QN, Offer SM, Diasio RB. Gene-specific variant classifier (DPYD-Varifier) to identify deleterious alleles of dihydropyrimidine dehydrogenase. Clin. Pharmacol. Ther. doi:10.1002/cpt.1020 (2018) (Epub ahead of print).

8 Boige $\mathrm{V}$, Vincent M, Alexandre P et al. DPYD genotyping to predict adverse events following treatment with fluorouracil-based adjuvant chemotherapy in patients with stage III colon cancer: a secondary analysis of the PETACC-8 randomized clinical trial. JAMA Oncol. 2(5), 655 (2016).

9 Lee AM, Shi Q, Pavey E et al. DPYD Variants as predictors of 5-fluorouracil toxicity in adjuvant colon cancer treatment (NCCTG N0147). J. Natl Cancer Inst. 106(12), 1-12 (2014).

10 Saam J, Critchfield GC, Hamilton SA, Roa BB, Wenstrup RJ, Kaldate RR. Commentary body surface area - based dosing of 5-fluoruracil results in extensive interindividual variability in 5-fluorouracil exposure in colorectal cancer patients on FOLFOX regimens. CLCC 10(3), 203-206 (2011).

11 Prado CMM, Baracos VE, McCargar LJ et al. Body composition as an independent determinant of 5-fluorouracil-based chemotherapy toxicity. Clin. Cancer Res. 13(11), 3264-3268 (2007).

12 Offer SM, Butterfield GL, Jerde CR, Fossum CC, Wegner NJ, Diasio RB. microRNAs miR-27a and miR-27b directly regulate liver dihydropyrimidine dehydrogenase expression through two conserved binding sites. Mol. Cancer Ther. 13(3), 742-751 (2014). 
13 Meulendijks D, Henricks LM, Amstutz U et al. rs895819 in mir27A improves the predictive value of DPYD variants to identify patients at risk of severe fluoropyrimidine-associated toxicity. Int. J. Cancer 138(11), 2752-2761 (2016).

14 Gamelin E, Delva R, Jacob J et al. Individual fluorouracil dose adjustment based on pharmacokinetic follow-up compared with conventional dosage: results of a multicenter randomized trial of patients with metastatic colorectal cancer. J. Clin. Oncol. 26(13), 2099-2105 (2008).

15 Lee JJ, Beumer JH, Chu E. Therapeutic drug monitoring of 5-fluorouracil. Cancer Chemother. Pharmacol. 78(3), 447-464 (2017).

16 Hamzic S, Kummer D, Milesi S et al. Novel genetic variants in carboxylesterase 1 predict severe early-onset capecitabine-related toxicity. Clin. Pharmacol. Ther. 102(5), 796-804 (2017).

17 Caronia D, Martin M, Sastre J et al. A polymorphism in the cytidine deaminase promoter predicts severe capecitabine-induced hand-foot syndrome. Clin. Cancer Res. 17(7), 2006-2013 (2011).

18 Meulendijks D, Henricks LM, Jacobs BAW et al. Pretreatment serum uracil concentration as a predictor of severe and fatal fluoropyrimidine-associated toxicity. Br. J. Cancer 116(11), 1415-1424 (2017).

19 Boisdron-celle M, Capitain O, Faroux R et al. Prevention of 5-fluorouracil-induced early severe toxicity by pre-therapeutic dihydropyrimidine dehydrogenase deficiency screening: assessment of a multiparametric approach. Semin. Oncol. 44, 13-23 (2017).

20 Van Staveren MC, Van Kuilenburg ABP, Guchelaar $\mathrm{H}$ et al. Evaluation of an oral uracil loading test to identify DPD-deficient patients using a limited sampling strategy. Br. J. Clin. Pharmacol. 81(3), 553-561 (2015). 